two aims should be co-ordinated to serve the needs of health care at all levels. A joint World Health Organisation International Committee for Standardisation in Haematology working group has now been set up on training and education programmes in haematology and will consider the laboratory service requirements for primary health care, clinics, and district hospitals. To promote the exchange of views and to have the problem defined by haematologists from developing countries, the International Committee for Standardisation in Haematology organised a symposium on the subject at the International Congress of Haematology in Montreal last year.

One of the contributions (see p 292) was a review by Dr Prawase Wasi and Dr Supa Na-Nakorn of the problems found in Thailand. Technical training and the practice of haematology in Bangkok are comparable with that anywhere in the world, with relatively many graduates, medical and technical, qualifying each year; but only a few miles away laboratory services are poor or almost non-existent, with few medical technologists in provincial hospitals and not a single trained technician in a district hospital. If the high standard of haematology training at the centre is to make an impact on health services elsewhere in the country, say Wasi and NaNakorn, appropriate training must be given to nurses and paramedical and auxiliary personnel to handle day-to-day haematological problems with a few simple tests.

After measurement of the haemoglobin concentration an intelligent appraisal of blood film morphology can often establish the cause of anaemia with no need for more complex tests. This requires visual comprehension rather than technical skill. How to teach this to laboratory aides with limited formal education was the theme of a paper by J R McArthur of Seattle. Lack of teachers makes it essential to use self-teaching material. The use of audiotape or a written manual linked to a set of $35 \mathrm{~mm}$ transparencies is an effective means for selfinstruction; its greatest value is that the student can work at his or her own pace. Programmes suitable for teaching morphology at all levels are available from various libraries, with a microfiche version as an alternative to slides. ${ }^{4}$ This has the advantage of being cost effective and space saving; and slides cannot be misplaced from the set. A videodisc format may be even more advantageous. The World Health Organisation has an important part to play in providing these selfteaching programmes adapted to the curricula and the educational levels of laboratory aides and technical assistants in developing countries.

Participants in the symposium from developing countries emphasised the fact that appropriate technology must not be an excuse for inferior technology. Account must be taken not only of ease of operation by relatively unskilled aides but also the need to ensure that the equipment is sufficiently accurate and also reliable in the field. The data need to be of good enough quality both for immediate clinical purposes and for analysis in epidemiological studies. The World Health Organisation has recognised as a priority the need for simple, inexpensive, battery-powered, robust, and relatively accurate equipment ${ }^{5}$-yet, despite the great potential demand for such equipment for the basic tests of haematology and clinical chemistry, little progress seems to have been made. One possible instrument for haemoglobinometry consists of a colorimeter using a light-emitting diode together with an ingenious method of automatic blood dilution from a disposable capillary tube, ${ }^{6}{ }^{7}$ but even this instrument is thought by some experts to be too complex to be useful under field conditions. The combination of cheapness, simplicity, and accuracy may not be attainable easily, either for haemo- globinometry or for any other clinical laboratory tests, but at least it should be a goal.

${ }^{1}$ McMinn A, Russell GJ. Training of medical laboratory technicians: $a$ handbook for tutors. Geneva: World Health Organisation, 1975.

2 Lèvy-Lambert E. Basic techniques for a medical laboratory: a manual for the training of laboratory personnel in developing countries. Geneva: World Health Organisation, 1974.

${ }^{3}$ Anonymous. Laboratory services at primary health care level. WHO Chron $1979 ; 33: 334-7$.

${ }^{4}$ American Society of Hematology. National slide bank catalogue of morphology slides, microfiche and teaching sets. Seattle: Health Sciences Learning Resources Centre, University of Washington, 1977.

5 World Health Organisation. Consultation on standardization in haematology. Bilthoven April/May 1965. Geneva: World Health Organisation, 1976. (Document LAB/75.3.)

${ }^{6}$ Pocock SN, Rideout JM. Short technical description of the MonA and PotI ab colorimeters. Fournal of Automatic Chemistry 1979;1 :222-3.

7 Nicholson KG, Metcalf CA, Renshaw A, Rideout JM. Simple method for measuring haemoglobin. Evaluation in Andean rain forest. Lancet 1977; i:836-7.

\section{Neurosyphilis again}

The decline of neurosyphilis in the postwar years has largely been due to the effective treatment of primary and secondary syphilis by penicillin, but it is also partly attributable to the inadvertent treatment of unrecognised early stages of the disease by antibiotics given for other, unrelated diseases. Younger physicians and psychiatrists may have had little experience of patients with neurosyphilis, and they may be in danger of overlooking "the most important infection of the nervous system encountered in psychiatric practice." ${ }^{1}$ Furthermore, inadequate treatment of primary and secondary syphilis may lead to unexpected late complications, as shown by a recent report ${ }^{2}$ of a man who had received 10 injections of penicillin for secondary syphilis but who evaded further treatment. Ten years later he presented with general paralysis of the insane; this was arrested by further treatment, but he remained demented.

The central nervous system is affected in $10 \%$ of primary and $30 \%$ of secondary syphilis, ${ }^{3}$ as evidenced by abnornal cerebrospinal fluid in the absence of symptoms and signs (early asymptomatic neurosyphilis); the response to adequate treatment is excellent provided any recurrent activity is detected by serial examinations of the blood and cerebrospinal fluid and further treatment given.

Meningovascular syphilis ${ }^{4}$ appears within five years of infection and causes acute or chronic spinal or cerebral leptomeningitis, Heubner's arteritis, and small meningeal gummata. Acute meningitis in the secondary stage mimics pyogenic infection and responds to penicillin. More common is a chronic meningitis producing headaches; palsies of the third, fourth, or fifth cranial nerves; and occasionally somnolence and diabetes insipidus from hypothalamic lesions. Disease of the cerebral convexities may cause fits, dementia, and focal signs, and hydrocephalus is an infrequent sequel to occlusion of the foramina by exudate. Gummata may present as tumours. Endarteritis is a more frequent complication, causing strokes from thrombosis of cerebral vessels-an important differential diagnosis of strokes in younger patients. Serological tests in the blood and cerebrospinal fluid are positive in $90 \%$ of cases.

Parenchymatous neurosyphilis embraces tabes, optic atrophy, syphilitic myelitis (amyotrophy), and general paralysis of the insane; only the last concerns us here, as a 
treatable cause of a wide variety of mental symptoms ranging from neurosis to psychosis and dementia. General paralysis devastated the French after the Napoleonic wars, and its syphilitic aetiology was slowly recognised as a result of the Wassermann reaction (1906) and the identification of Treponema pallidum in the brain by Noguchi and Moore (1913). ${ }^{5}$ The meninges are thickened, the cortex is atrophic with perivascular lymphocytes, and microglial rod cells, in palisades, give a granular appearance to the ependyma and cortex. General paralysis starts insidiously 10 to 15 years after the primary infection, with fatigue, irritability, and forgetfulness. Depression, sexual excesses, and boastfulness supervene, with alcohol intolerance and defects of judgment heralding dementia. Motor signs follow: tremulous speech, a wavy tremor of the lips, transient hemiparesis, and later a spastic pyramidal paresis; impotence and loss of libido are the final ironies. Argyll Robertson pupils are present in most but not all patients and may appear late. Serological tests are positive in untreated patients, and a lymphocytosis $\left(10\right.$ to $\left.100 \times 10^{3} / 1\right)$ and raised protein concentrations $(0.5$ to $1.2 \mathrm{~g} / \mathrm{l})$ in the cerebrospinal fluid are invariable signs of infection of the central nervous system; a positive Lange curve is an outdated index of raised globulin and IgG concentrations, but a positive result to the VDRL test and specific antibodies may persist after treatment.

Differential diagnosis is not difficult provided that the possibility is borne in mind. Grandiose forms, simple dementia, depressive illness, and taboparesis are recognised presenting syndromes, but the earliest symptoms of neuroticism and irascibility, or of fits and "cerebral ischaemic episodes" may escape detection. Psychiatrists of the past picked out paranoid, neurasthenic, and acute organic pictures, so that virtually any psychiatric illness can be mimicked. Lishman's recommendation "that all patients admitted to psychiatric units should have serological tests carried out" 1 is sound, and needs emphasising when these eminently treatable syndromes are diminishing.

Diligent search will discover occasional patients with asymptomatic (latent) neurosyphilis-an abnormal cerebrospinal fluid in the absence of symptoms and signs. Falsepositive reactions need to be excluded by the more specific antibody tests for $T$ pallidum and by raised cell and protein values. Untreated general paralysis of the insane is fatal within one to five years, and to be effective the treatment of all forms of neurosyphilis should be given early and in full doses (usually penicillin 1 megaunit daily, by intramuscular injection for 20 days $^{6}$ ), and followed by further examinations of the cerebrospinal fluid for at least two years. Relapses need to be recognised, treated, and checked until neither the cell count nor the protein concentration in the cerebrospinal fluid is raised. Herxheimer reactions (fever, fits, and exacerbations of signs) occur in 5\% of patients in the first few days. Penicillin sensitivity may be circumvented by treatment with tetracycline $2 \mathrm{~g}$ daily for 20 days or erythromycin, though the long-term efficacy of these drugs is less certain. Primary resistance of spirochaetes is still uncommon, but reports of persisting organisms $^{7}$ after apparently full courses of treatment make continued vigilance essential.

${ }^{1}$ Lishman WAL. Organic psychiatry. Oxford: Blackwell, 1978:388-406.

${ }^{2}$ Hoffman BF. Neurosyphilis in a young man. Can $\mathcal{F}$ Psychiatry $1981 ; 26$ : 68-70.

${ }^{3}$ Hahn RD, Clark EG. Asymptomatic neurosyphilis: a review of the literature. American Fournal of Syphilis, Gonorrhea and Venereal Diseases $1946 ; 30: 305-16$.

${ }^{4}$ Elliott FA. Clinical neurology. Philadelphia: WB Saunders, 1971 :316-8.

5 Noguchi H, Moore JW. A demonstration of Treponema pallidum in the brain in cases of general paralysis. F Exp Med 1913;17:232-8.

6 Csonka G. Venereal diseases. Medicine (Oxford) 1972; No 6:502-13.

7 Anonymous. Persistence of treponemes after treatment of syphilis. Lancet $1965 ; \mathrm{i}: 693-4$.

\section{Pharmacists as doctors}

The appetite of the British public for-and its ability to absorb-opinion and advice about medical matters could never fully be met by the resources available in general practice. Alternative sources include grannies, agony columnists, and publicans at one end of the range, with over-thecounter prescribing by pharmacists at the other.

Advice by pharmacists has perhaps for too long been assumed to lie outside the clinical, legal, and educational conventions of traditional medical practice. No one seems to know how much of pharmacists' interaction with their clients represents prescribing on the advice of the pharmacist as against the sale of preparations requested by the customer. What is clear is that the increasing professionalism and "professionalisation" of pharmacists have highlighted a series of awkward questions. If pharmacists are to develop their function as prescribers in the increasingly complex medical arena what training should they have in clinical medicine? Should-and can-guidelines for prescribing practice be laid down? Should clinical records be kept, because sooner or later actions taken by pharmacists are going to be questioned, with consequent risks of litigation? And which, if any, parts of prescribing can safely be delegated to less trained counter assistants? Answers to some of the questions were provided earlier this year when a substantial part of one issue of the Pharmaceutical fournal ${ }^{1}$ was devoted to the report of a working party of the Council of the Pharmaceutical Society set up "to prepare guidance to be given in both undergraduate and postgraduate courses on the response to symptoms described in general practice."

On the positive side, the prescribing guidelines suggested in the report are both sensible and reassuring-as would have been expected from a broadly based working party that included respected representatives of the medical and pharmaceutical professions. Other welcome features were the importance placed by the report on the need for pharmacists to learn interviewing skills and to understand the importance of knowledge of local industries, illnesses, and idioms as well as to recognise that what patients complain of may be a remarkably misleading guide to their real problems. Courses for pharmacy students must, as the report implies, reflect their need to learn something of the general practitioner's skills in consulting with patients. Ideally, this learning ought to be based on tuition similar to what medical students (and in some areas nurses and social work students) increasingly receive in teaching general practices. This could be yet another sound reason for encouraging the growth of properly staffed and financed teaching practices.

At the same time as welcoming these initiatives by our pharmaceutical colleagues we should pause to ask ourselves as doctors whether some of the problems raised by the report have become problems because of the constraints and structures of modern general practice. Patients have more difficulty in seeing a general practitioner than some general practitioners like to think, and those who have most difficulty in coping with the system are often those who most need professional help. Probably unwittingly, the generally negative message of waiting room posters creates the feeling that doctors are there to see patients only when all else fails, but even the best booklets on self-care have to be supplemented by some professional opinion.

${ }^{1}$ Anonymous. Response to symptoms in general practice pharmacy. Pharmaceuticalfournal $1981 ; 226: 14-8$. 